\title{
Variación espacio-temporal en la composición de macroalgas en la zona intermareal rocosa en Salango, provincia de Manabí, Ecuador
}

\section{Spatio-temporal variation in the macroalgae composition in the rocky intertidal zone in Salango, Manabi province, Ecuador}

\section{César Sánchez ${ }^{1 *} \&$ Genoveva Torres²}

\author{
1 Interagua, laboratório de Aguas-Ecuador \\ ${ }^{2}$ Instituto de Investigaciones de Recursos Naturales, Facultad de Ciencias Naturales, \\ IIRN, Avenida Juan Tanca Marengo s/n Avenida Las Aguas
}

Recibido 10 de abril 2021; recibido en forma revisada 2 de mayo 2021, aceptado 4 de junio 2021 Disponible en línea 26 de junio 2021

\begin{abstract}
Resumen
En el presente trabajo se determinó la variación espaciotemporal en la composición de macroalgas en dos sitios de muestreo localizados en Salango, provincia de Manabí, en la costa de Ecuador. Se registraron las variaciones de los grupos taxonómicos, cobertura y diversidad en función del tiempo. La metodología empleada es la de SARCE y el manual de métodos de ecosistemas marinocostero para establecer impactos ambientales. Entre los resultados, Centroceras clavulatum es la especie que presenta el mayor registro en ambas localidades muestreadas. La mayor cobertura en la estación sur está representada por Chlorophyta (37\%), mientras que, en el norte se registró una mayor cobertura de Rhodophyta (39\%). Según el índice de Shannon-Wiener e valor máximo de diversidad se dio en noviembre para el norte y sur de Salango 2,1 y 2,3 respectivamente. La variación temporal, demostró que los grupos de Rhodophyta y Chlorophyta presentan cambios en cuanto al número de individuos entre los meses de octubre y noviembre del 2019, Los índices de diversidad presentaron niveles elevados en noviembre. Los valores de similitud coinciden con la diversidad de especies y riqueza de especies en el mes de noviembre donde se obtuvieron los valores de salinidad más altos sugiriendo que sus poblaciones poseen una serie de variaciones espaciales, pero no tienen una dinámica paralela y cada grupo atiende a diferentes requerimientos para su población.
\end{abstract}

Palabras claves: cobertura, diversidad, macroalgas, variación.

\begin{abstract}
In this work, the space-time variation in macroalgae composition was determined at two sampling sites located in Salango, Manabí province, on the coast of Ecuador. Variations in taxonomic groups, coverage and diversity were recorded over time. The methodology used is sarCE and the marine-coastal ecosystem method manual for establishing environmental impacts. Among the results, Centroceras clavulatum is the species with the largest record in both sampled locations. The largest coverage in the southern station is represented by Chlorophyta (37\%), while in the north there was a higher coverage of Rhodophyta (39\%). According to the Shannon-Wiener index and maximum diversity value was given in November for the north and south of Salango 2.1 and 2.3 respectively. Temporary variation showed that The Rhodophyta and Chlorophyta groups have changes in the number of individuals between October and November 2019, Diversity rates had high levels in November. The similarity values coincide with species diversity and species richness in November where the highest salinity values were obtained by suggesting that their populations have a number of spatial variations, but do not have parallel dynamics and each group meets different requirements for its population.
\end{abstract}

Keywords: coverage, diversity, macroalgae, variation. 


\section{Introducción}

Las macroalgas son la fuente de la productividad primaria, constituyen la base de la red trófica para los ecosistemas marino-costeros, también contribuyen en la formación de grandes cantidades de materia orgánica que pasan a formar parte de la cadena alimentaria. Son componentes de diferentes ambientes en donde proporcionan nutrientes, hábitat, refugio y zonas de asentamiento larval, además tienen la facultad de asentarse en distintos sustratos arenosos, arrecifes de coral, litorales rocosos y manglares, adoptando diferentes tipos de morfología en función de la complejidad de su estructura y adaptaciones al medio (Carr, 1991; Cordero, 2016).

Los sustratos rocosos en las zonas intermareales forman uno de los principales hábitats para el asentamiento de las macroalgas, su distribución en estos sustratos depende de los factores abióticos y su interacción con el medio, tales como el nivel de la marea, la influencia del oleaje y los diferentes tipos de roca. Los diversos tipos de sustratos pueden incluir plataformas, acantilados, bloques y cantos rodados (Cordero, 2016; Garcia, Acosta, Londoño-Cruz, \& Cantera, 2012; Manzanos, 2014).

La flora de la Zona Intermareal Rocosa (ZIR) está constituida por algas rojas (Rhodophyta), verdes (Chlorophyta) y pardas (Phaeophyta), con esta interactúa una fauna asociada constituida en su mayoría por distintas especies de invertebrados marinos. La zona intermareal se divide en tres áreas: (i) la zona supralitoral, en donde solo recibe la humedad por aspersión o "rocío" del mar; (ii) la zona mesolitoral, está cubierta durante el periodo de pleamar, pero permanece expuesta en condiciones de bajamar; y, (iii) la zona infra litoral, sumergida por largos periodos, excepto durante el ciclo donde la marea alcanza su máxima bajamar. En cada zona, las condiciones ambientales tanto físicas (desecación, temperatura), como químicas (salinidad, concentración de oxígeno) y nutrientes varían por los periodos de transición de la marea (INVEMAR, 2004; Menge \& Branch, 2001; Vinueza \& Flores, 2002).

Las comunidades de macroalgas y los distintos grupos de organismos que están asociadas a los ZIR, son de vital importancia desde un punto de vista ecológico y científico. Desde la perspectiva de la ecología han demostrado ser indispensable para el funcionamiento y desarrollo de los distintos ecosistemas en las zonas costeras, en igual forma todas estas comunidades operan a diversas escalas espaciales y temporales influyendo en la riqueza específica como lo es la biodiversidad (Carr, 1991; Jover, Llorente, \& Viña, 2009).

Las playas de la parroquia Salango presentan dos zonas con características de litorales rocosos. Estas áreas están influenciadas por el turismo y la constante pesca artesanal e industrial, siendo esta última un posible productor de distintos contaminantes dirigidos hacia el mar, debido a su relación directa con el litoral que los rodea. Además, la falta de estudios en el área provee una serie de vacíos en el conocimiento y comportamiento de las especies de flora en la zona. Los grupos algales responden a los cambios en el ambiente modificando su riqueza y abundancia en el ecosistema, particularmente algunos grupos morfofuncionales están asociados a cambios en las variables físicas, como la sedimentación y la entrada de nutrientes.

Las macroalgas suelen responder a cambios en el ecosistema lo que puede modificar su abundancia, incluso la presencia o ausencia de algunas especies dependen de cambios en variables ambientales. Por lo tanto, el presente trabajo planea determinar la variación espaciotemporal en la composición de macroalgas en la zona intermarial rocosa de Salango.

\section{Antecedentes}

Los estudios realizados en el Ecuador sobre macroalgas inician en 1835 en las islas Galápagos con W.H. Honey, cuando identifica una variante del género de las coralinas de tipo incrustante. William Randolph Taylor como parte de la expedición de Allan Hancock en el Pacífico, realizó en 1945 la primera caracterización sobre la flora algal de las islas y algunos puntos del continente, donde reportó 35 especies de chlorophyta, 34 especies de phaeophyta y 137 especies de rhodophyta. En 1988, Gary Kendrick realizó una recopilación de algas marinas bénticas en las costas de Ecuador, en la cual se describen 320 especies donde se indica el endemismo y sus hábitats característicos. En 1974, Flor de María Valverde realizó una publicación denominada: “vegetación marina de Ballenita, península de Santa Elena Ecuador - Provincia del Guayas. El Instituto Nacional de Pesca, en 1994 realizó el proyecto de investigación científica "algas marinas del Ecuador", donde se recopila información y se reporta nuevas distribuciones de las macroalgas regionales. Posteriormente, se publicó el primer estudio ecológico-ficológico denominada "Estudio ecológico de Rhodophytas de las provincias del Guayas y Manabí” (Müller \& Salazar, 1996).

La mayoría de las publicaciones sobre el estudio de ficología fueron realizados en la reserva marina de las Galápagos (R.M.G.) en conjunto con la fundación Charles Darwin (F.C.D.), los resultados de estas investigaciones formaron una "línea base" reportando 316 especies de algas marinas y 36 taxones para Galápagos, con un endemismo del 29\% (Garske, 2002). Existen estudios ficológicos realizados en el Ecuador continental en distintas estaciones y meses del año, como lo realizado en la provincia de Santa Elena mediante un análisis de la distribución, diversidad y abundancia de macroalgas en la zona intermareal rocosa, donde se identificaron una total de 21 especies, de éstas, 9 corresponden a la división chlorophyta, 8 rhodophyta y 4 heterocontophyta (Rubira, 2012).

Dentro de este marco en años posteriores se realizó en la provincia de Manabí , un estudio sobre la variación espacio-temporal de grupos algales en dos ecosistemas 
de fondo rocoso (submareal), donde se presentan diferentes niveles de disturbios antropogénicos, además, las unidades de muestreo fueron evaluadas en términos de los grupos morfofuncionales algales y éstos constituyeron dos grupos generales: macroalgas y "turf algae. o "césped”, estos últimos poseen una altura menor a $2 \mathrm{~cm}$ (Betancourtt, 2015).

Actualmente, en el Ecuador continental las investigaciones sobre el estado actual referente a la ecología de macroalgas en las zonas litorales son muy escasas, a diferencia de los realizados en las islas Galápagos, donde existe una mayor cantidad de trabajos dirigidos a investigación y conservación.

\section{Materiales y métodos}

\section{Área de estudio}

La localidad de Salango se ubica en el cantón Puerto López, en la Provincia de Manabí, en la costa Ecuador. Es una población rural que cuenta con una superficie de $88 \mathrm{~km}^{2}$. Al suroeste se ubica la isla Salango, esta posee aproximadamente 2 hectáreas de extensión. La parroquia está influenciada por la cuenca hidrográfica del río Ayampe, donde escurre durante todo el año para esta población, además en el área se encuentran el río Salango y estero río chico, ambos son intermitentes durante la época invernal y de forma gradual se vacían durante la estación seca
(Cuenca \& Eliecer, 2014; GAD Cantón Puerto López, 2015) (Fig. 1).

\section{Fase de Campo}

El presente trabajo se llevó según la metodología propuesta por SARCE (The South American Research Group on Coastal Ecosystems, 2012), Para el establecimiento de un mejor diseño de investigación se incluyó como referencia al "Manual de métodos de ecosistemas marino-costero para establecer impactos ambientales de Colombia" (INVEMAR, 2014).

El estudio se realizó en el periodo octubre - diciembre del 2019, y enero del 2020, el muestreo fue de cada 15 días según los periodos de bajamar, los cuales se obtuvieron por medio de las tablas de mareas del INOCAR (Instituto Oceanográfico y Antártico de la Armada del Ecuador), y se fijó a Puerto López como referencia del nivel de mar en Salango.

Se establecieron dos estaciones de muestreo que corresponden a las zonas norte y sur en el área rocosa de la playa. En cada estación se realizó un transecto lineal de 10 metros perpendicular a la costa dejando un metro de espacio para cada cuadrante (Fig. 2).

Para determinar la cobertura algal se utilizó el método de intersección en cuadriculas para organismos sésiles (SARCE, 2010) se trabajó de manera perpendicular

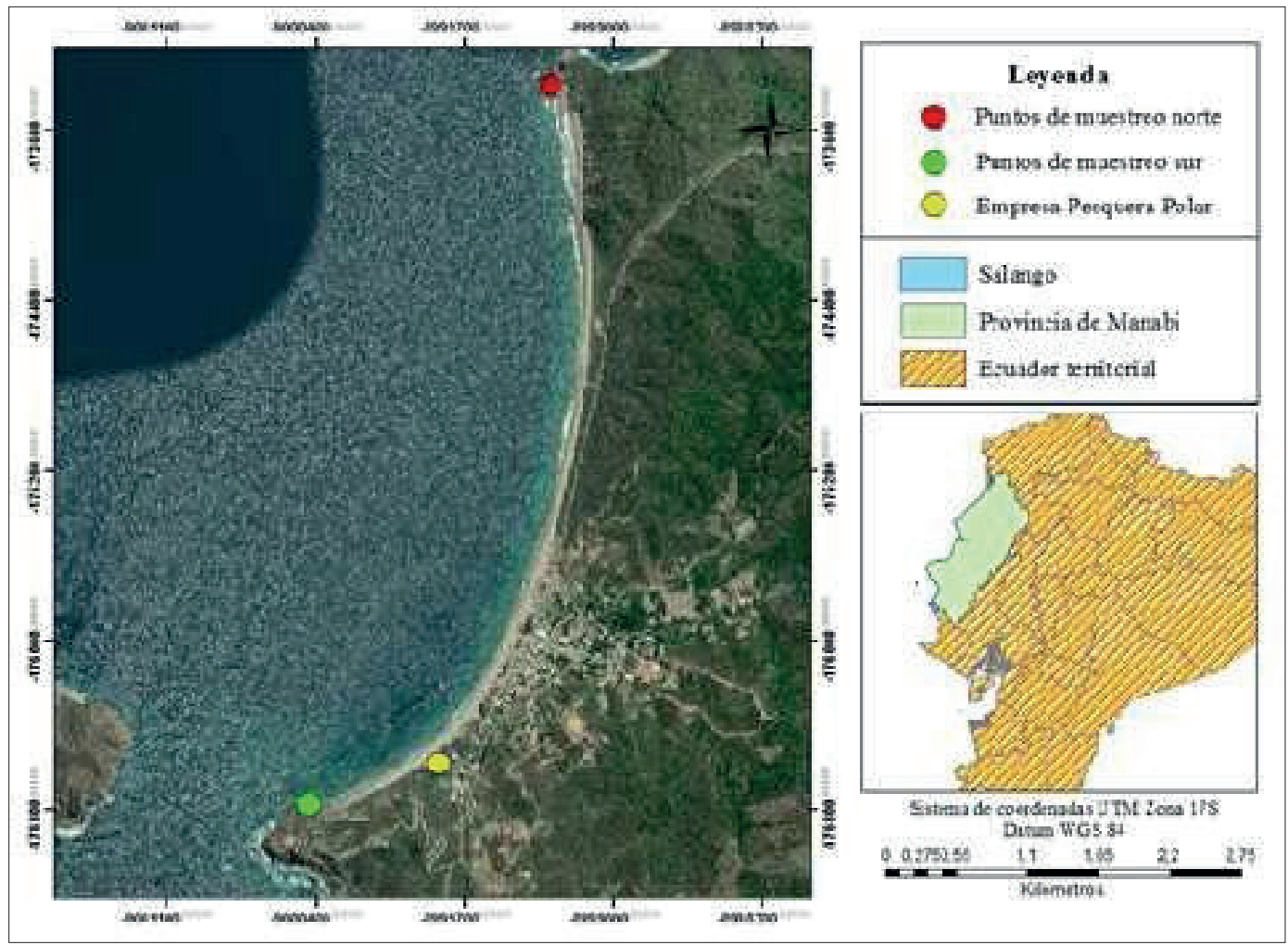

Figura 1. Sitos de recolección de muestras, parroquia Salango, cantón Puerto López, provincia de Manabí- Ecuador. 


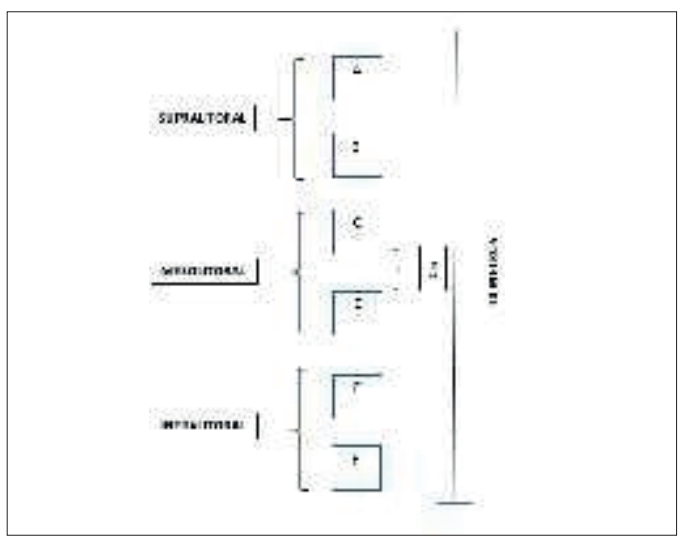

Figura 2. Método de muestreo; con sus respectivo transecto y cuadrantes en la zona intermareal rocosa en Salango Manabí Ecuador

a la costa con una totalidad de seis (6) cuadrantes sumados de $1 \mathrm{~m} 2$ (24 cuadrantes de $50 \mathrm{~cm} \times 50 \mathrm{~cm}$ ) (Fig. 3).

Para la colecta de muestras de macroalgas en las distintas zonas, se utilizaron fundas de cierre hermético y la extracción de las macroalgas se realizó con ayuda de un cuchillo curvo, para preservar las muestras fueron transportadas en un cooler para su posterior análisis en el laboratorio también, se tomaron los datos de salinidad con ayuda de un refractómetro.

Cada transecto fue georreferenciado mediante la aplicación de "Maps" proporcionado por Google LLC para equipos Android (Tabla 1), Todas las coordenadas de fueron registradas en sistema UTM (Universal Transverse Mercator) (Tabla 1).

La composición se determinó mediante la identificación y presencia de macroalgas (Li-Alfaro \& Zafra-Trelles, 2012) según los cuadrantes a lo largo del transecto in situ en la zona intermareal rocosa y el procesamiento de las muestras en el laboratorio.'

\section{Fase de laboratorio}

Una vez terminada la colecta las muestras fueron transportadas hacia el laboratorio del instituto de investigaciones de recursos naturales (IIRN), ubicado en la facultad de Ciencias Naturales, donde se lavaron con abundante agua y pasaron a un proceso de tamizado, separando arena, piedras y

\begin{tabular}{cccc}
$\begin{array}{c}\text { Estaciones de } \\
\text { Muestreos }\end{array}$ & $\begin{array}{c}\text { Sitios de } \\
\text { muestreos }\end{array}$ & Latitud & Longitud \\
\hline SUR & A & 1.761 .111 & 80.841 .802 \\
NORTE & B & 1.598 .247 & 80.851 .955 \\
\hline
\end{tabular}

Tabla 1. Coordenadas de localización de los transeptos y sitios de muestreo en la parroquia Salango.

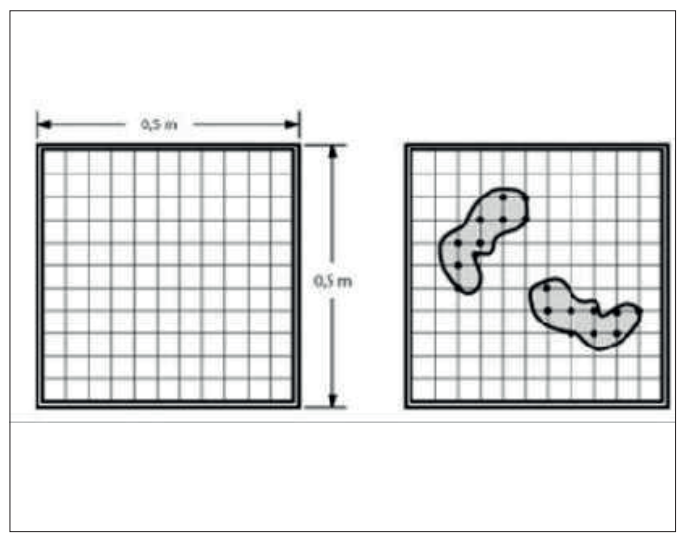

Figura 3. El porcentaje de cobertura es igual al número intersecciones en una cuadricula de 100 intersecciones, imagen modificada de (SARCE, 2010).

macroinvertebrados, con un estereomicroscopio las macroalgas fueron separadas e identificadas hasta el más bajo nivel taxonómico con ayuda de bibliografía especializada (FAO, 1995; Müller \& Salazar, 1996; Smith, 1966; Taylor, 1967), posteriormente las muestras fueron rotuladas, clasificadas y preservadas con formol al $4 \%$.

\section{Análisis de datos}

Para cada estación y meses de muestreos se determinó la Riqueza Especifica (S) el cual, es obtenido por el número total de especies en el muestreo de la comunidad.

Se estimó la diversidad en cada área de muestreo, mediante el índice de Shannon utilizando con ayuda del software estadístico Past versión 3.20 para medir la diversidad del área, cuyos valores inferiores a dos son considerados diversidad baja y superiores a tres diversidades altas de especies (León, 2018).

$$
H^{\prime}=-\sum_{\bar{i}=1}^{S} p i \log _{2} p i
$$

$\boldsymbol{S}=$ Número de especies (la riqueza de especies).

$\boldsymbol{p} \boldsymbol{i}=$ Porción de individuos de la especie $\boldsymbol{i}$ respecto al total de individuos. (Es decir, la abundancia relativa de la especie $i$ ): $\frac{n i}{N}$

$\boldsymbol{n}_{\mathbf{i}}=$ Número de individuos de la especie $i$.

$\mathbf{V}=$ Numero de todos los individuos de todas las especies.

Para expresar el grado de semejanza entre las comunidades de macroalgas en las estaciones norte y sur según sus especies, se utilizó el coeficiente de similitud de Sorensen:

$$
I S=2 c \frac{2 c}{(a+b)} * 100
$$


Para determinar la distribución de las especies en el tiempo se utilizó elÍndice de Constancia (Bodenheimer, 1955):

$$
C=\frac{P m * 100}{P M}
$$

Donde:

$$
\begin{aligned}
& \boldsymbol{P} \boldsymbol{m} \text { : Número de meses en que se reporta la especie } \\
& \boldsymbol{P M} \text { : Número total de meses muestreados para determinar la } \\
& \text { distribución de las especies en el tiempo }
\end{aligned}
$$

Para conocer la distribución espacial se empleó el índice de Frecuencia (Bodenheimer, 1955):

$$
F r=\frac{P \text { 릐 } 100}{P H}
$$

Donde:

$\boldsymbol{P} \boldsymbol{h}$ : Número de estaciones en que se reporta la especie

$\boldsymbol{P H}$ : Número total de estaciones de muestreo.

\section{Resultados}

Cualitativamente fueron registradas en la zona litoral rocosa en las dos estaciones un total de 12 especies de macroalgas, para la clase Chlorophyta se registraron 4 especies: (i) Boodlea composita, (ii) Ulva lactuca, (iii) Cladophora sp., (ix) Enteromorpha sp. En la clase de las Rhodophyta se identificaron 7 especies: (i) Centroceras clavulatum, (ii) Hypnea sp, (iii) Jania longiarthra, (ix) Chondracanthus saundersii, (x) Lophosiphonia sp., (xi) Corallina sp., (xii) Gelidium sclerophyllum y para Phaeophyta: Padina sp. (i) (Tabla 2).

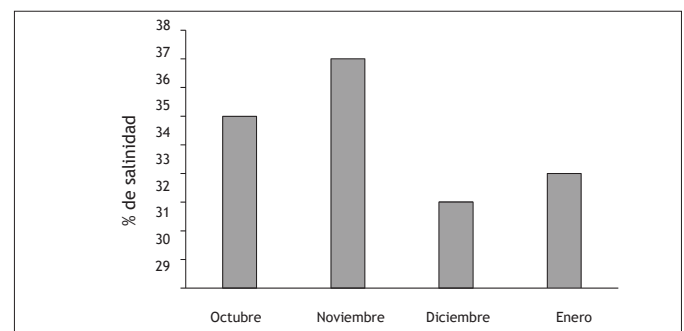

Figura 4. Valores de salinidad reportados para la zona intermareal rocosa de Salango.

La salinidad cambio durante el periodo de muestreo, sin embargo en el mes de noviembre se obtuvo el valor más alto (Fig. 4).

El porcentaje de cobertura en la zona intermareal rocosa a nivel de división demostró que en la estación Sur las Chlorophytas tenían una mayor cobertura mientras que en el norte quien tuvo el valor más alto fueron las Rodhohytas en ambas estaciones se evidencio una ausencia de macroalgas representado también en este análisis y que varía entre las estaciones durante el periodo octubre 2019 - enero 2020 (Fig. 5).

El índice de Shannon-Weaver indicó que en la parte sur el mayor valor de diversidad se dio en noviembre $\left(H^{\prime}=2.3\right)$ y el más bajo en enero $\left(H^{\prime}=1.6\right)$, en el

\begin{tabular}{|c|c|c|c|c|c|}
\hline \multirow[b]{2}{*}{ Phylum } & \multirow[b]{2}{*}{ Orden } & \multirow[b]{2}{*}{ Familia } & \multirow[b]{2}{*}{ Nombre Científico } & \multicolumn{2}{|c|}{ No. de indiv } \\
\hline & & & & Sur & Norte \\
\hline \multirow{4}{*}{ Clorophyta } & \multirow{2}{*}{ Cladophorales } & Boodleaceae & Boodlea composita & 8 & 6 \\
\hline & & Cladophoraceae & Cladophora sp. & 7 & 8 \\
\hline & \multirow{2}{*}{ Ulvales } & \multirow{2}{*}{ Ulvaceae } & Ulva lactuca & 10 & 8 \\
\hline & & & Enteromorpha sp. & 5 & 2 \\
\hline \multirow{7}{*}{ Rodophyta } & \multirow{2}{*}{ Ceramiales } & Ceramiaceae & Centroceras clavulatum & 10 & 10 \\
\hline & & Rhodomelaceae & Lophosiphonia sp. & 4 & 1 \\
\hline & \multirow{2}{*}{ Corallinales } & \multirow{2}{*}{ Corallinaceae } & Jania longiarthra & 8 & 8 \\
\hline & & & Corallina sp. & 2 & 2 \\
\hline & \multirow{2}{*}{ Gigartinales } & Cystocloniaceae & Hypnea sp. & 9 & 10 \\
\hline & & Gigartinaceae & $\begin{array}{c}\text { Chondracanthus } \\
\text { saundersii }\end{array}$ & 4 & 2 \\
\hline & Gelidiales & Gelidiaceae & Gelidium sclerophyllum & 3 & 4 \\
\hline Phaeophyta & Dictyotales & Dictyotaceae & Padina sp. & 2 & 0 \\
\hline
\end{tabular}
norte se presentaron valores altos durante el mes de noviembre $\left(\mathrm{H}^{\prime}=2.14\right.$ y $\left.\mathrm{H}^{\prime}=2.13\right)$ y los bajos en Octubre y diciembre ( $\left.\mathrm{H}^{\prime}=1.7\right)$ (Fig. 6).

Tabla 2. Macroalgas presentes en la zona intermarial rocosa en las zonas sur y norte de Salango. 


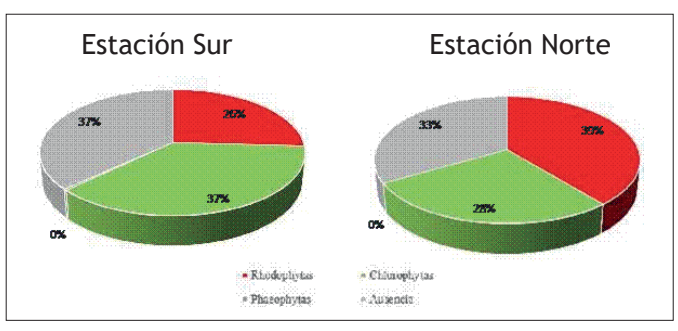

Figura 5. Representación la cobertura algal en ambas estaciones de Salango.

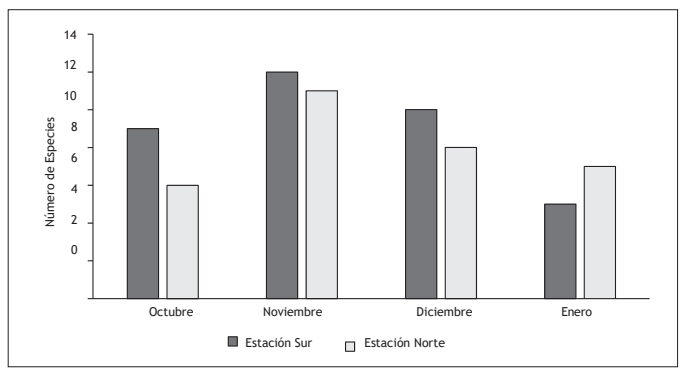

Figura 7. Variaciones espaciotemporales de la riqueza especifica de las macroalgas en la zona litoral rocosa Salango.

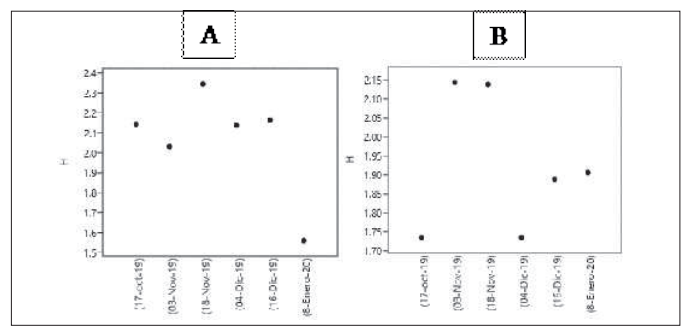

Figura 6. Valores del índice de Shannon-Weaver durante el periodo octubre 2019 a enero 2020 para el sur (A) y norte (B) de Salango.

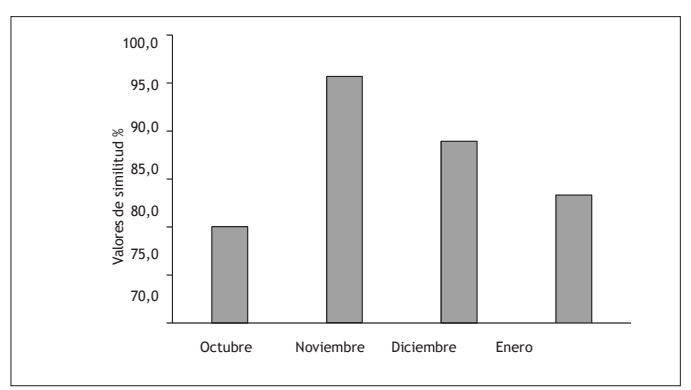

Figura 8. Variación temporal del índice de similitud de Sorensen de la zona litoral rocosa Salango.

Tabla 3. Lista de especies y valores de frecuencia y constancia de macroalgas del litoral rocoso de Salango. Fr = Frecuencia y $\mathrm{C}=$ Constancia.

\begin{tabular}{|c|c|c|c|c|c|c|c|c|c|c|}
\hline \multirow{3}{*}{$\begin{array}{l}\text { Listado de } \\
\text { Especies }\end{array}$} & \multicolumn{8}{|c|}{ Estaciones de muestreo } & \multirow{3}{*}{ C. } & \multirow{3}{*}{ Fr. } \\
\hline & \multicolumn{4}{|c|}{ SUR } & \multicolumn{4}{|c|}{ NORTE } & & \\
\hline & Oct & Nov & Dic & Ene & Oct & Nov & Dic. & Ene & & \\
\hline \multicolumn{11}{|l|}{ División } \\
\hline \multicolumn{11}{|l|}{ Clorophyta } \\
\hline Enteromorpha sp. & $x$ & $x$ & $x$ & $x$ & & $x$ & & $x$ & 75 & 100 \\
\hline Boodlea composita & $x$ & $x$ & $x$ & & $x$ & $x$ & $x$ & $x$ & 100 & 100 \\
\hline Ulva lactuca & $x$ & $x$ & $x$ & $x$ & $x$ & $x$ & $x$ & $x$ & 100 & 100 \\
\hline Cladophora sp. & & $x$ & $x$ & $x$ & & $x$ & $x$ & $x$ & 100 & 100 \\
\hline \multicolumn{10}{|l|}{ División } & \\
\hline Centroceras clavulatum & $x$ & $x$ & $x$ & $x$ & $x$ & $x$ & $x$ & $x$ & 100 & 100 \\
\hline Hypnea sp. & $x$ & $x$ & $x$ & $x$ & $x$ & $x$ & $x$ & $x$ & 75 & 100 \\
\hline \multirow[t]{2}{*}{$\begin{array}{c}\text { Jania longiarthra } \\
\text { Chondracanthus } \\
\text { saundersii }\end{array}$} & $x$ & $x$ & $x$ & & $x$ & $x$ & $x$ & $x$ & 50 & 100 \\
\hline & & $x$ & $x$ & & & $x$ & $x$ & & 50 & 100 \\
\hline Lophosiphonia sp. & & $x$ & $x$ & & & $x$ & & & 75 & 100 \\
\hline Amphiroa sp. & $x$ & $x$ & & & $x$ & $x$ & & & 50 & 100 \\
\hline Gelidium sclerophyllum & & $x$ & $x$ & & & $x$ & $x$ & & 50 & 100 \\
\hline \multicolumn{11}{|l|}{ División } \\
\hline \multicolumn{11}{|l|}{ Phaeophyta } \\
\hline Padina sp. & $x$ & $x$ & & & & & & & 50 & 50 \\
\hline
\end{tabular}


El valor más alto de la riqueza especifica de encontró en la estación sur en noviembre con 12 especies y el más bajo en la misma estación durante el mes de enero, en general, la riqueza especifica presento una ligera variación durante los meses de muestreo, en ambas estaciones los mayores valores de riqueza se registraron en el mes de noviembre. Los valores más bajos se obtuvieron en el mes de enero 2020 (Fig. 7).

El coeficiente de similitud de Sorensen presento valores altos durante los meses de muestreo demostrando que existe un mayor grado de similitud entre estaciones (Fig. 8).

Las especies, Hypnea Sp., Ulva lactuca, Cladophora Sp., Centroceras clavulatum Sp., Boodlea composita, Jania longiarthra, Enteromorpha Sp., presentaron una distribución amplia en las dos estaciones de muestreo en la zona intermareal rocosa de Salango. Sin embargo Chondracanthus saundersii, Lophosiphonia sp, Amphiroa Sp, Gelidium sclerophyllum, Padina $s p$ presentaron una distribución media, en ambas estaciones. Las especies que se presentaron constantemente durante los cuatro meses de muestreo fueron, U. lactuca, Cladophora Sp., C. clavulatum, Hypnea Sp. sin embargo, Padina sp. Solo se la encontró en la estación sur (Tabla 3).

\section{Discusión}

La riqueza de especies de macroalgas en la región intermareal de Salango es alta en los dos puntos de muestreos teniendo una leve variación, probablemente se deba a la composición del sustrato, según Gino (2012) el suelo rocoso permite una mayor capacidad de albergar una gran diversidad de macroalgas lo que coincide con Sole \& Beatriz, (1997) en su caracterización de macroalgas bénticas en Venezuela. La variación de la diversidad se explica por los factores abióticos como oleaje, radiación, temperatura entre otros, que pueden influir en la presencia y ausencia de las macroalgas (DelgadilloGarzón \& Newmark, 2008; Edding, Tala, \& Vásquez, 2006; Espinoza-Avalos, 2005; Quirós- Rodriguez, Arias- Rios, \& Ruiz, 2016), además, la influencia y exposición a fuentes directas de nutrientes puede ser aprovechado por especies oportunistas disminuyendo la diversidad en un ecosistema (Li-Alfaro \& ZafraTrelles, 2012) lo que podría explicar la variación en el mes de enero.

La composición macroalgal de la playa el Salango está caracterizada por la presencia de especies conspicuas del género Ulvales, Cystocloniaceae y Ceramiales, se conoce que estos géneros son tolerantes a un amplio rango de factores ambientales de salinidad y temperatura y elevados niveles de nutrientes (Candelaria, Vargas, López, \& González-González, 2006; Dante C., Gil, \& Juscamaita, 2011; Garduño, 2019).

En la zona litoral rocosa encontramos especies que toleran la desecación como U. latuca, es probable que sea por tener membranas celulares coriáceas (Jover et al., 2009; PÉREZ, 2019), sin embargo, el área de muestreo es un ambiente, de oleaje y turbulencia casi constantes lo que no permite una acumulación de nutrientes por lo que su presencia no podría ser oportunista.

Los valores de similitud coinciden con la diversidad de especies y riqueza de especies en el mes de noviembre donde se obtuvieron los valores de salinidad más altos, según la salinidad influye en procesos metabólicos de las algas como el crecimiento creando un ambiente favorable para ellas (Areces \& Araujo, 1996; DelgadilloGarzón \& Newmark, 2008).

\section{Conclusión}

Existen pocos trabajos disponibles para el estudio de las macroalgas, el presente es una contribución somera sobre el comportamiento de las poblaciones algales, en la zona costera de Salango.

-La composición de macroalgas es similar en ambos puntos de muestreo durante el periodo octubre 2019 - enero 2020.

En las dos estaciones en Salango se colectaron 12 especies de macroalgas, entre éstas, C. clavulatum, $U$. Lactuca e Hypnea sp. , presentaron el mayor registro en cuanto a la variación de especies.

Las Clorophytas son las macroalgas que predominan en el lado sur de Salango según a cobertura algal cerca a actividades antropogénicas, mientras que en el norte son las Rodophytas.

Los índices de diversidad, riqueza específica y similaridad representaron valores más altos en noviembre coincidiendo con la elevada salinidad reportada en los dos puntos de muestreo, sin embargo, no se tomaron otros aspectos ambientales para afirmar este enunciado.

\section{Recomendaciones}

- Investigaciones previas para este tipo de trabajos, donde exista un registro o línea base en la caracterización de macroalgas a nivel de especie.

- El registro de temperatura superficial, fotoperiodo, el grado de composición de la arena sobre los litorales rocosos deben ser obtenidos en futuras investigaciones.

- $\quad$ Se sugiere el análisis datos de, pH y O.D. para corroborar que la presencia de macroalgas en las distintas zonas.

- Incrementar el espacio para cada transecto a y extender el periodo de estudio para tener una mejor representatividad en el área 


\section{Bibliografía}

Areces, A. J., \& Araujo, M. (1996). Influencia de la salinidad y la temperatura sobre el crecimiento de Bryothamnion triquetrum (Rhodophyta: Rhodomelaceae). Revista de Biologia Tropical, 44(2), 449-454.

Betancourtt, C., Zapata, J., Latorre, N., Anguita, C., Castañeda, F., Meynard, A.,

... Contreras-Porcia, L. (2018). Variación espacio-temporal en la composición del ensamble de macroalgas del intermareal rocoso de Maitencillo, Valparaíso, costa central de Chile. Revista de Biología Marina y Oceanografía, 53(1), 105-117. https: //doi.org/10.4067/s0718-

19572018000100105

Bodenheimer, F. S. (1955). PRECIS D'ECOLOGIE ANIMALE. (B. S. Payot, Ed.)

(1st ed.). italia.

Candelaria, C. F., Vargas, D., López, N., \& González-González, J. (2006). PATRÓN DE DISTRIBUCIÓN DE MACROALGAS EN UN CANAL DE CORRIENTES, 65-72.

Carr, M. H. (1991). Habitat selection and recruitment of an assemblage of temperate zone reef fishes, 146, 113-137.

Cordero, M. (2016). Caracterización ficológica del litoral rocoso y manglares de yapascua, parque nacional san esteban, estado carabobo. universidad de carabobo.

Cuenca, F., \& Eliecer, T. (2014). Modelo de desarrollo turístico sustentable para el fortalecimiento económico de la comuna Salango del cantón Puerto López-Provincia de Manabí. Repositorio Institucional UPAO.

Dante C., A. , Gil, P. , \& Juscamaita, J. (2011). Estabilización de la "Marea Verde" causada por Ulva lactuca (Ulvophyceae, Chlorophyta) a través del ensilaje. Anales Científicos, 72(1), 13-18. https: / / doi.org/10.21704/ac.v72i1.853

Delgadillo-Garzón, O., \& Newmark, F. (2008). Cultivo piloto de macroalgas rojas (rhodophyta) en bahía portete, la guajira, Colombia. Boletin de Investigaciones Marinas y Costeras, 37(2), 7-26.

Edding, M., Tala, F., \& Vásquez, J. (2006). Fotosíntesis, productividad y algas marinas, (january), 1-39.

Espinoza-Avalos, J. (2005). Fenología de macroalgas marinas. Hidrobiológica, 15(1), 109-122.

FAO. (1995). Guia fao para la identificion de especies para los fines de la pesca.

Roma.

Garcia, S., Acosta, A., Londoño-Cruz, E., \& Cantera, J. (2012). Organismos sésiles y móviles del litoral rocoso en el Pacífico Colombiano:Una guía visual para su identificación. INVEMAR Series de Documentos Especialesno. 26. Garduño, A.(2019). Análisis morfológico, molecular y filogenético del género Centroceraskützing (Rhodophyta ,Ceramiaceae ) en el Golfo de Méxicoy mar Caribe mexicano.

Garske, L. E. (2002). Macroalgas MARINAS. In E. Danulat \& E. Graham (Eds.), Reserva Marina de Galápagos. Línea Base de la Biodiversidad (pp. 426-429).

INVEMAR. (2004). Informe del Estado de los Ambientes Marinos y Costeros en Colombia. Panamericana Formas e Impresos 2005.

INVEMAR. (2014). Manual De Métodos De Ecosistemas Marinos Y Costeros Con Miras a Establecer Impactos Ambientales. Colombia.

Jover, A., Llorente, G., \& Viña, N. (2009). Variación espaciotemporal de la composición de macroalgas del mesolitoral rocosos del sector Aguadores, Plataforma Suroriental, Cuba. Revista de Investigaciones Marinas, 30(1), 3- 9.

León, A. (2018). Distribución espacial de macroinvertebrados bentónicos en el intermareal rocoso de la punta de san lorenzo, santa elena, ecuador.

Li-Alfaro, G., \& Zafra-Trelles, A. (2012). Composición, abundancia y diversidad de macroalgas en el litoral de puerto Malabrigo, La Libertad - Perú 2009. Sciéndo, 15(1),
33-42.

LOPEZ, G. P. (2015). Plan de desarrollo y de ordenamiento terriorial Puerto López. Puerto López.

Manzanos, E. R. (2014). Clasificación física del intermareal rocoso y distribución de macroalgas a diferentes escalas espaciales a lo largo del NEAtlántico.

Menge, B., \& Branch, G. (2001). Rocky intertidal communities. In B. M, G. S, \&

H. M (Eds.), Marine Community Ecology (p. 550).

Müller, H. , \& Salazar, M. (1996). algas Marinas del Ecuador (1er ed.). Guayaquil: Comision Asesora Ambiental - Instituto Nacional de pesca.

PÉREZ, D. (2019). Incremento de nitrógeno en el tejido de la macroalga oportunista ulva lactuca (linnaeus), asociado a ondas internas de marea. I, 53(tecnology), 8-17.

Quirós- Rodriguez, J., Arias- Rios, J. E., \& Ruiz, R. (2016). Estructura de las comunidades macroalgales asociadas al litoral rocosodel departamento de córdoba, colombia, 32(2), 339-354.

RUBIRA CARVACHE, K. (2012). Diversidad, Abundancia Y Distribución De Las Macroalgas En La Zona Intermare Al Rocoso En Las Pla Yas De Salinas, La Libertad Y Ballenita (Península De Santa Elena - Ecuador Octubre - Noviembre 2009 ), 92. $\quad$ Retrieved from http:// repositorio.ug.edu.ec/bitstream/redug/713/1/ DIVERSIDAD\%2C abundancia y distribución de las macroalgas en la zona intermareal rocoso en las playas de salinas\%2c la libertad y ballenita \%28península de santa elena - ecuador o.pdf

SARCE. (2010). Protocol and sampling design for marine diversity and biomass assessments for the South American research group on coastal ecosystems.

Smith, G. (1966). Marine algae of the monterrey peninsula california.

Sole, M., \& Beatriz, V. (1997). Caracterizacion de las macroalgas marinas benticas en la region chirimena-punta caiman, do. Miranda, Venezuela. Sciences-New York, 33(3), 180-190.

Taylor, R. (1967). Géneros de algas marinhas de la Costa Atlántica Latinoamericana.

Vinueza, L., \& Flores, M. (2002). Comunidades intermareales rocosas. In E. Danulat \& E. Graham (Eds.), Reserva Marina de las Galápagos. Línea Base de la Biodiversidad (pp. 98 114). Santa Cruz- Ecuador. 